\title{
CO OBSERVATIONS OF CANDIDATES FOR CARBON-RICH ASYMPTOTIC GIANT BRANCH AND POST-ASYMPTOTIC GIANT BRANCH STARS
}

\author{
KeVIN VOLK AND SUN KwOK \\ Department of Physics and Astronomy, The University of Calgary, Calgary, Alberta, Canada T2N 1N4 \\ AND \\ ANDREW W. WOODSWORTH \\ Dominion Astrophysical Observatory, National Research Council, 5071 West Saanich Road, Victoria, BC, Canada V8X 4M6 \\ Received 1992 February 26; accepted 1992 July 9
}

\begin{abstract}
Circumstellar $\mathrm{CO}$ emission has been detected in a number of featureless or weak $\mathrm{SiC}$ emission, low color temperature IRAS sources. The $\mathrm{CO}$ detections confirm the suggestion that these are either extreme carbon stars, carbon-rich proto-planetary nebulae (PPNs), or carbon-rich planetary nebulae (PNs). We find that the $\mathrm{CO}$ emission is relatively stronger for a given luminosity in post-AGB stars than AGB stars, suggesting a more efficient excitation mechanism is at work in the post-AGB phase. One probable post-AGB star was detected in $\mathrm{HCN}$ for the first time. The available $\mathrm{HCN}$ data suggest a rapid decline in $\mathrm{HCN}$ emission after the AGB. Molecular emission is shown to be a useful tracer of the evolution from asymptotic giant branch to the planetary nebula phase.

Subject headings: circumstellar matter — infrared: stars — stars: carbon stars: post-asymptotic giant branch
\end{abstract}

\section{INTRODUCTION}

Stars on the asymptotic giant branch (AGB) can generally be separated into two classes: oxygen-rich (M stars) or carbonrich (C stars) based on the oxygen to carbon abundance ratio. Some AGB stars have $\mathrm{C}: \mathrm{O}$ abundance ratios close to 1 and are spectroscopically classified as $\mathbf{S}$ stars. It is traditionally assumed that $\mathbf{S}$ stars are in transition from $\mathbf{M}$ to $\mathbf{C}$ stars, but this hypothesis is never demonstrated observationally or theoretically. It is, however, firmly established that stars on the AGB begin as O-rich, and some (probably not all) later develop into $\mathrm{C}$ stars as the result of carbon dredge-up from the core (Iben \& Renzini 1983). As stars ascend the AGB, they develop increasingly strong stellar winds. Most evolved AGB stars are heavily obscured by dust and are discovered as the result of infrared surveys. The AFGL survey covered the midinfrared region and discovered infrared sources with color temperatures as low as $300 \mathrm{~K}$. The $I R A S$ survey extended the wavelength coverage to $100 \mu \mathrm{m}$ and detected sources of much lower temperatures. It is possible that some of these lowtemperature sources correspond to stars that have very thick dust shells and are near the end of AGB evolution.

Although the photospheric composition cannot be directly observed for these late AGB stars, their O- or C-rich nature can be determined through the circumstellar dust features. Oxygen-rich stars show the $9.7 \mu \mathrm{m}$ silicate feature (in emission or absorption), and C-fich stars show the $11.3 \mu \mathrm{m} \mathrm{SiC}$ feature or a featureless continuum (cf. Kwok 1987). The properties of evolved O-rich stars have been extensively studied, for they often show $\mathrm{OH}$ maser emission and are known as $\mathrm{OH} / \mathrm{IR}$ stars (Herman \& Habing 1987). The most extreme O-rich stars, meaning those with the highest mass-loss rates and reddest colors, are easily identified by their deep silicate absorption features (Kwok, Hrivnak, \& Boreiko 1987).

However, the properties of extreme $C$-rich stars are less well known. The best studied extreme carbon stars are CW Leo $\left(\right.$ IRC $\left.+10^{\circ} 216\right)$ and AFGL 3068. The infrared continua of both objects have low color temperatures, suggesting that they have very thick circumstellar envelopes. $\mathrm{CW}$ Leo has a weak $\mathrm{SiC}$ feature, probably as the result of self-absorption in the highly optically thick dust envelope. AFGL 3068 shows an almost featureless continuum between 10 and $20 \mu \mathrm{m}$. These two examples therefore raise the possibility that the most extreme C-rich stars may have no strong circumstellar dust features and can be identified only by their infrared colors.

A search of extreme carbon stars using the IRAS LowResolution Spectrometer (LRS) data has recently been performed by Volk, Kwok, \& Langill (1992, hereafter Paper I). Thirty-one candidates have been identified with IRAS colors similar to CW Leo and AFGL 3068, but which show only a weak SiC feature or no feature at all in the 7-23 $\mu \mathrm{m}$ LRS range. Candidates for which near-infrared photometric data are available show a blackbody-like energy distribution, with color temperatures in the range of 300-450 K. Most of these candidates have no known associations with existing astronomical catalogs other than the AFGL catalog. Their color and spectral similarity to $\mathrm{CW}$ Leo and AFGL 3068 suggest that these are probably extreme carbon stars (hereafter referred as group 1).

There also exists another group of infrared sources that are featureless in the mid-infrared, but have color temperatures lower than those of the above group, typically $200 \mathrm{~K}$ (hereafter referred to as group 2). The LRS spectra of 12 sources with these properties are plotted in Figure 1. The LRS spectra were extracted and processed at the University of Calgary IRAS Data Analysis Facility (cf. Volk et al. 1991). Although the nature of these low color temperature sources is not certain, it is likely that they have evolutionary connections to extreme carbon stars, probably C-rich proto-planetary nebulae (PPNs), or C-rich planetary nebulae (PNs). We have plotted the color distribution of these two groups of C-rich objects in Figure 2. We can see that the two groups are clearly separated in IRAS colors. 

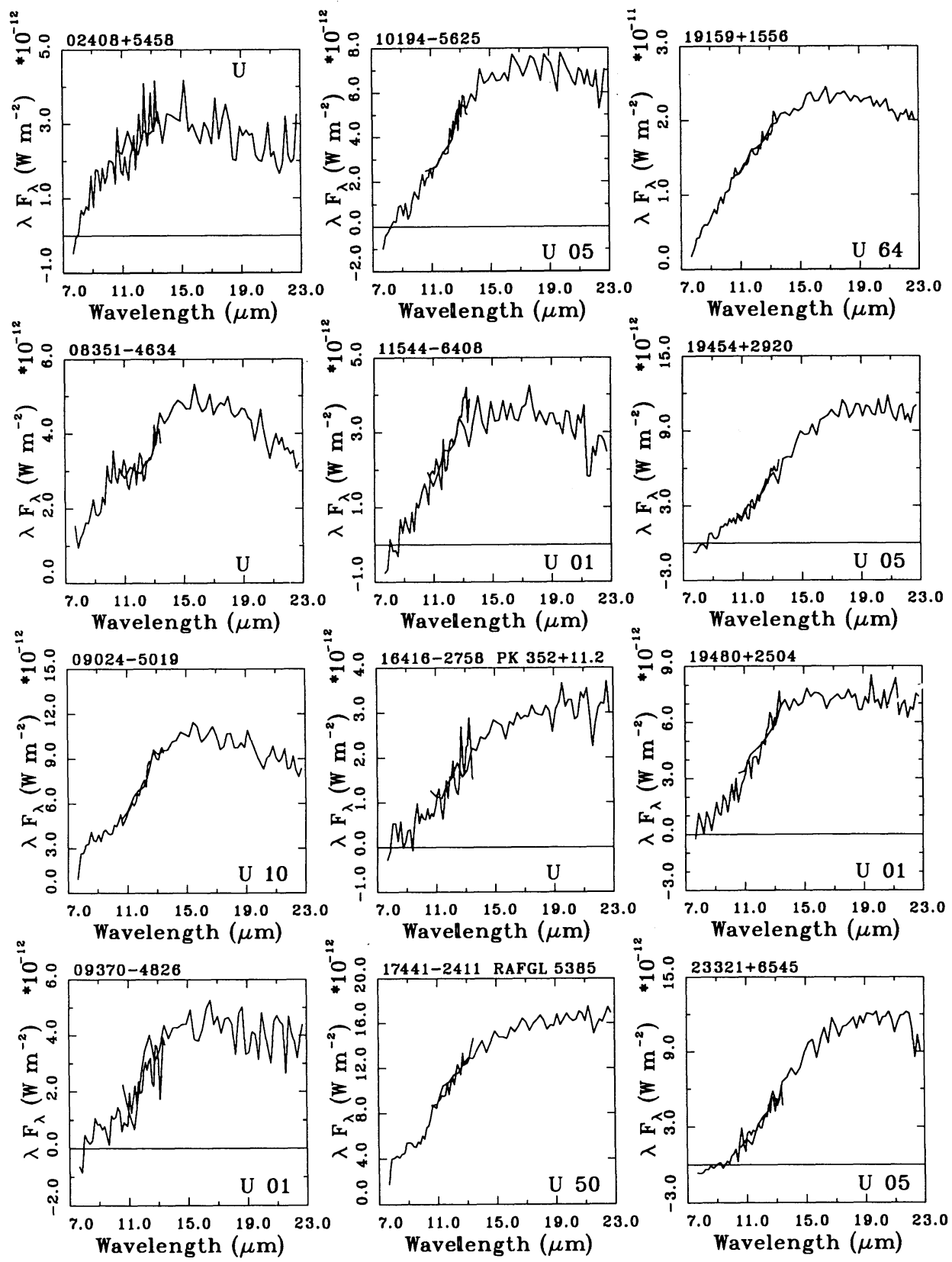

FIG. 1.-The LRS spectra of 12 low color temperature featureless IRAS sources. Other catalog associations (when present) are listed next to the IRAS names. The letter code U refers to the spectral classification code of Volk \& Cohen (1989), and the two-digit spectral classification codes are those of the LRS Atlas.

The nature of the above two groups of low color temperature, featureless continuum objects can only be exactly determined by infrared spectroscopy. However, because of the sharp dropoff in the energy distribution shortward of $10 \mu \mathrm{m}$, this is not easy to undertake. While highly evolved $\mathrm{C}$ stars do not have the unique signature of $\mathrm{OH}$ maser emission as in O-rich stars, they should all show strong optically thick thermal CO emission (Knapp et al. 1982). Highly evolved C stars are expected to have flat tops (resolved) or parabolic (unresolved) profiles and velocity widths of $\sim 30 \mathrm{~km} \mathrm{~s}^{-1}$ which distinguish them from $\mathrm{CO}$ emissions from interstellar molecular clouds. $\mathrm{CO}$ emission from O-rich stars appears to be $\sim 3$ times weaker than for $\mathrm{C}$ stars when other factors are equal (Knapp \& Morris 1985). If some of these objects are PPNs or young PNs, CO emission should still be present for the remnant circumstellar envelopes. Of the total of 43 objects in these two groups, 27 are located north of declination $-30^{\circ}$ and are accessible to a northern millimeter wavelength telescope. In this paper, we report a survey of these two classes of objects in $\mathrm{CO}$ and HCN using the James Clerk Maxwell Telescope.

\section{OBSERVATIONS}

The observations were carried out on the first shifts of the nights of 1991 October 25-27, using the $15 \mathrm{~m}$ James Clerk Maxwell Telescope located on Mauna Kea, Hawaii. The telescope beam size at $230 \mathrm{GHz}$ is $22^{\prime \prime}$, which is well suited for stellar observations. The $\mathrm{CO}$ observations were carried out under the dual-polarization mode, which reduces the observ- 


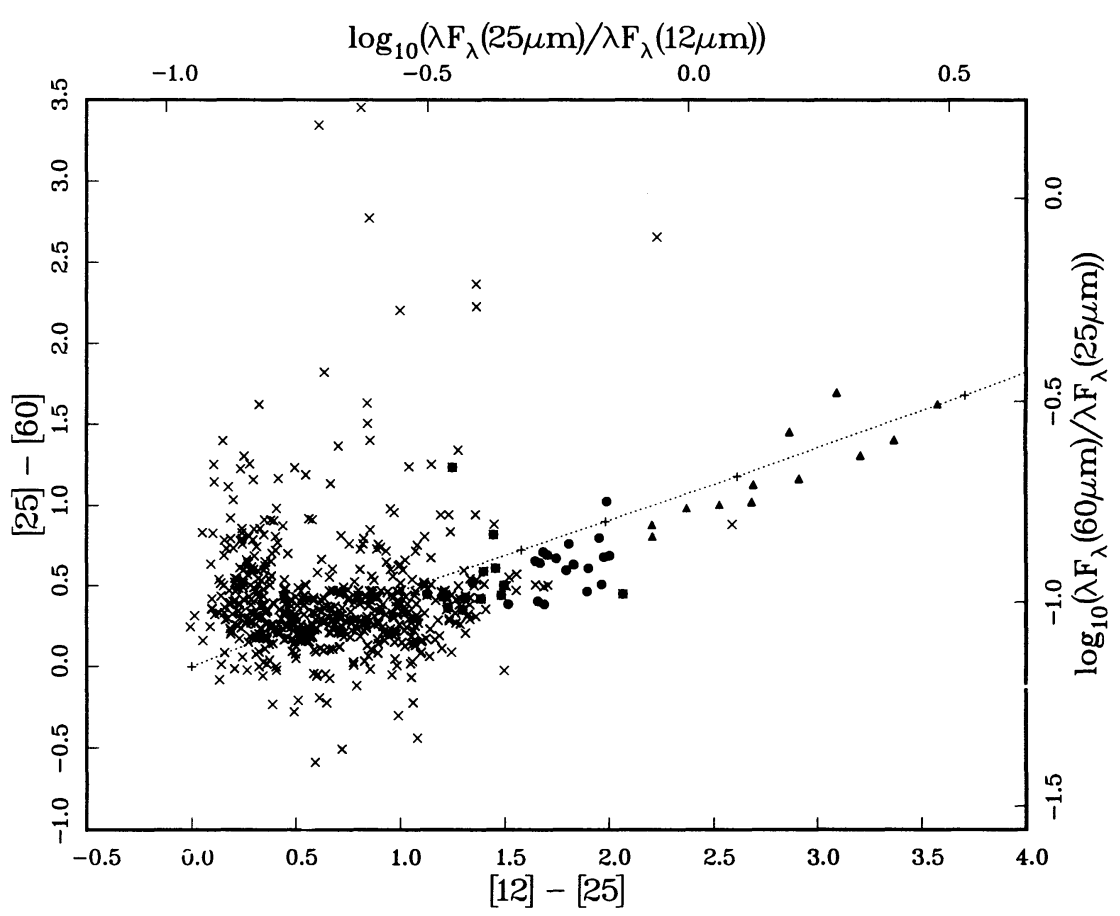

FIG. 2.-Distribution of the group 1 and group 2 carbon-rich objects in the IRAS color-color diagram. Group 1 sources are shown as filled circles, and group 2 sources as filled triangles. Also plotted are the 564 stars (crosses) in the LRS Atlas that are classified as group C (11.3 $\mu \mathrm{m}$ emission; cf. Volk et al. 1991). The blackbody curve is shown as a dotted line. The IRAS colors are as defined by Walker \& Cohen (1988).

ing time by a factor of 2 for an unpolarized source. The 2048 channel acousto-optical spectrometer (AOSC) was used for the back end. The channel spacing is $250 \mathrm{KHz}$, which corresponds to an effective resolution of $330 \mathrm{kHz}$, or a velocity resolution of $0.44 \mathrm{~km} \mathrm{~s}^{-1}$ at $230 \mathrm{GHz}$. In the dual-polarization mode, the frequency shift was set to about 1000 channels, so the 2048 spectrometer channels were organized into two 1024 channel spectra. The processing software later averaged the two polarizations, optically scaling them by some factor to account for gain differences in the two IF paths. The $\mathrm{CO}$ rest frequency was set at $230.538 \mathrm{GHz}$.

The observing procedure involving chopping against a cold load, and alternating on- and off-source measurements. The time at each position was generally $60 \mathrm{~s}$. For the spectral observations a chopper beam throw of $3^{\prime}$ in azimuth and a chopping frequency of $1 \mathrm{~Hz}$ were used. The typical integration time per source was 40 minutes. The telescope pointing and focus were initially set by observing Uranus and were rechecked several times during the night by observing compact $\mathrm{H}$ II regions taken from a list of suitable pointing targets. The changes in pointing were typically $2^{\prime \prime}-3^{\prime \prime}$, much smaller than the telescope beamwidth.

The scans were analyzed at the telescope using the SPECX package to combine the two polarizations of each scan and then to average all the scans for each object. The line widths, peak values, and integrated intensities were found using the SPECX software.

Three of the program sources were also observed in the $265.8864 \mathrm{GHz} H C N(J=3 \rightarrow 2)$ line on October 27. For these higher frequency observations the dual-polarization method could not be used and normal spectral observations were carried out. The typical system temperatures for October 25 and 26 were $\sim 900 \mathrm{~K}$ at $230 \mathrm{GHz}$. On October 27 , the weather conditions were worse and the system temperature was about $1350 \mathrm{~K}$ at $230 \mathrm{GHz}$ and $3000 \mathrm{~K}$ at $266 \mathrm{GHz}$.

The program sources initially included all of the sources from Paper I with R.A. between 19 and 07. Several sources were excluded because they had already been observed (see Zuckerman \& Dyck 1986a and references therein) in both CO $(J=1 \rightarrow 0)$ and $(J=2 \rightarrow 1)$. AFGL 3068 was observed for comparison, although it has been well observed in the past. In addition, four group 2 sources with R.A. from 19 to 24 were included in the program. The young, C-rich PN IRAS $21282+5050$ was also observed for comparison, because some of the extreme carbon star candidates may be evolving towards the PN phase (those with faint optical counterparts; see Paper I) and because the low-temperature sources may be PNs. IRAS $21282+5050$ was detected in ${ }^{12} \mathrm{CO}$ and ${ }^{13} \mathrm{CO}$ by Likkel et al. (1988). They also detected $\mathrm{HCO}^{+}$but not $\mathrm{H}_{2} \mathrm{O}, \mathrm{HCN}, \mathrm{OH}$, $\mathrm{SO}_{2}$, or $\mathrm{H}_{2} \mathrm{~S}$.

\section{RESULTS}

The results of the observations are shown in Figure 3 for all of the detections plus the HCN nondetection for IRAS $21282+5050$. In all cases the measured antenna temperature $T_{A}^{*}$ is used. The radiation temperature $T_{r}^{*}$ can be obtained from $T_{A}^{*}$ by dividing by the forward-scattering beam efficiency ( $\eta$ ) which we take to be 0.7 at $230 \mathrm{GHz}$ (Matthews 1991). The measured line parameters for all the sources are listed in Table 1 . The table gives the line peak $T_{A}^{*}$, the integrated line intensity, the line center LSR velocity $\left(V_{1 s r}\right)$ and the envelope expansion velocity $\left(V_{e}\right)$. IRAS $19480+2504$ was only observed for 20 minutes at high airmass, so the quality of the data for this source is poorer than for any of the others. Only two of the sources were not detected in CO: IRAS $19238+1159$ from the 

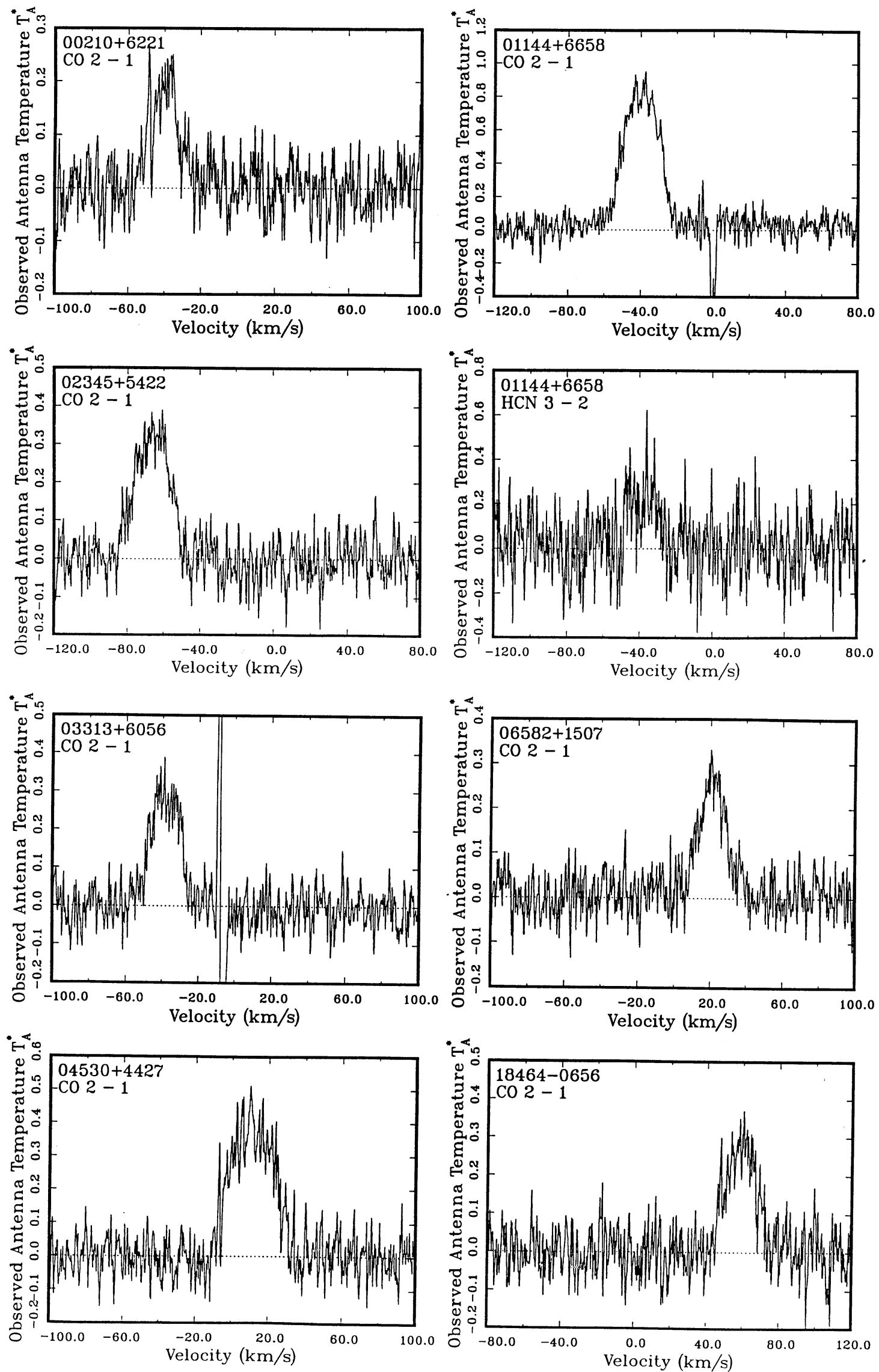

FiG. 3.-The CO (2-1) and HCN (3-2) spectra for the 17 detected sources, plus the nondetection of HCN (3-2) in IRAS $21282+5050$, after baseline subtraction and the combining of individual observations with the SPECX software. 



FIG. 3-Continued

296 



Fig. 3-Continued

TABLE 1

Observed Parameters

\begin{tabular}{|c|c|c|c|c|c|}
\hline IRAS Name & Line & $\begin{array}{l}\text { Peak } T_{A}^{*} \\
(\mathrm{~K})\end{array}$ & $\underset{\left(\mathrm{K} \mathrm{km} \mathrm{s}{ }^{-1}\right)}{T_{A}^{*} d V}$ & $\underset{\left(\mathrm{km} \mathrm{s}^{-1}\right)}{V_{\mathrm{LSR}}}$ & $\begin{array}{c}V_{e} \\
\left(\mathrm{~km} \mathrm{~s}^{-1}\right)\end{array}$ \\
\hline $00210+6221 .$. & $\mathrm{CO}$ & 0.20 & 3.7 & -39.9 & 16.7 \\
\hline $01144+6658 .$. & $\mathrm{CO}$ & 0.77 & 19.0 & -40.0 & 19.6 \\
\hline $02345+5422$. & $\mathrm{CO}$ & 0.31 & 7.7 & -67.0 & 19.0 \\
\hline $03313+6058$ & $\mathrm{CO}$ & 0.27 & 5.8 & -38.3 & 13.9 \\
\hline $04530+4427 \ldots \ldots \ldots \ldots$ & $\mathrm{CO}$ & 0.35 & 11.2 & +10.7 & 20.9 \\
\hline $06582+1507 \ldots \ldots \ldots \ldots$ & $\mathrm{CO}$ & 0.28 & 5.1 & +24.0 & 16.6 \\
\hline $18464-0656 \ldots$ & $\mathrm{CO}$ & 0.27 & 5.7 & +59.3 & 16.7 \\
\hline $19075+0921 \ldots \ldots \ldots \ldots$ & $\mathrm{CO}$ & 0.43 & 16.0 & +38.2 & 21.6 \\
\hline $19159+1556 \ldots \ldots \ldots \ldots$ & $\mathrm{CO}$ & $<0.15$ & $\ldots$ & $\ldots$ & $\ldots$ \\
\hline $19238+1159 \ldots \ldots \ldots \ldots$ & $\mathrm{CO}$ & $<0.15$ & & $\ldots$ & \\
\hline $19454+2920 \ldots \ldots \ldots \ldots$ & $\mathrm{CO}$ & 0.21 & 3.8 & +19.7 & 17.8 \\
\hline $19480+2504 \ldots \ldots \ldots \ldots$ & $\mathrm{CO}$ & 0.17 & 5.8 & +42.0 & 27.0 \\
\hline $19594+4047 \ldots \ldots \ldots \ldots$ & $\mathrm{CO}$ & 0.56 & 17.1 & +22.1 & 19.6 \\
\hline $21282+5050 \ldots \ldots \ldots \ldots$ & $\mathrm{CO}$ & 3.01 & 65.1 & +15.7 & 19.8 \\
\hline $21218+5631 \ldots \ldots \ldots \ldots$ & $\mathrm{CO}$ & 0.89 & 20.6 & +1.7 & 19.6 \\
\hline $21489+5301 \ldots \ldots \ldots \ldots$ & $\mathrm{CO}$ & 0.54 & 17.1 & -27.2 & 26.7 \\
\hline $22303+5950 \ldots \ldots \ldots \ldots$ & $\mathrm{CO}$ & 0.22 & 6.0 & -66.9 & 20.6 \\
\hline $23166+1655 \ldots \ldots \ldots \ldots$ & $\mathrm{CO}$ & 2.03 & 40.2 & -31.7 & 15.7 \\
\hline $23321+6545 \ldots \ldots \ldots \ldots$ & $\mathrm{CO}$ & 0.37 & 7.1 & -55.3 & 18.2 \\
\hline $01144+6658 .$. & $\mathrm{HCN}$ & 0.23 & 4.6 & -37.6 & 14.2 \\
\hline $21282+5050 \ldots \ldots \ldots \ldots$ & $\mathrm{HCN}$ & $<0.2$ & & & \\
\hline $23166+1655 \ldots \ldots \ldots \ldots$ & $\mathrm{HCN}$ & 0.93 & 16.0 & -29.6 & 12.4 \\
\hline
\end{tabular}




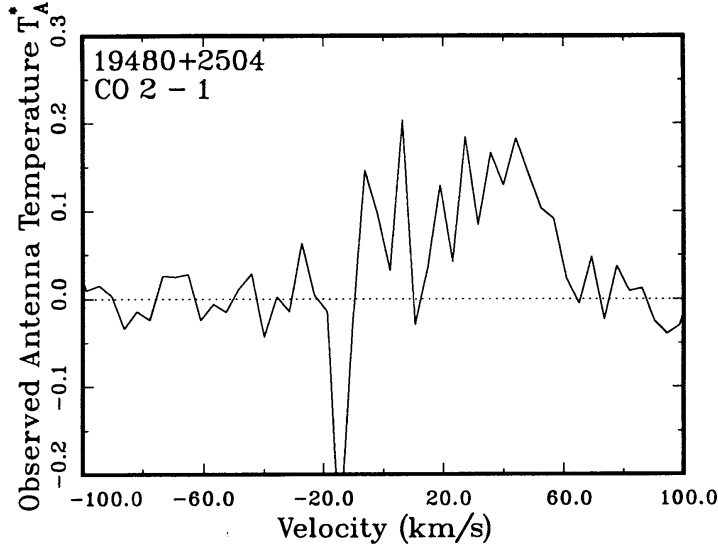

Fig. 4.-The CO spectrum of IRAS $19480+2504$ after averaging over 10 channels.

extreme carbon star candidates and IRAS $19159+1556$ from the low-temperature sources.

For the two sources for which $\mathrm{CO}$ was not detected, the upper limits are estimated from the noise level. For IRAS $19480+2504$ the detection is marginal, so we show the spectrum binned over 10 channels in Figure 4. There is some interstellar CO absorption in the reference beam for this source, which causes a distortion of the lower velocity part of the profile. Due to the poorer quality of the spectrum, the derived values for this source are less accurate than for the other sources.

Three of the brightest CO sources (AFGL 3068, IRAS $21282+5050$, and IRAS $01144+6658)$ were also observed in the $\operatorname{HCN}(J=3 \rightarrow 2)$ line. The integration time was 30 minutes per source. AFGL 3068 was clearly detected, IRAS $01144+6658$ was detected with poor signal-to-noise ratio, and IRAS $21282+5050$ was not detected. The nondetection of HCN $(J=3 \rightarrow 2)$ in IRAS $21282+5050$ is consistent with the nondetection of $\mathrm{HCN}(J=1 \rightarrow 0)$ by Likkel et al. (1988). HCN $(J=1 \rightarrow 0)$ was detected in IRAS $01144+6658$ by Zuckerman
\& Dyck (1986a), and in AFGL 3068 by Sopka et al. (1989). None of these sources has been previously observed in HCN $(J=3 \rightarrow 2)$.

\subsection{Derived Mass-Loss Rates}

All of the sources which have been detected have parabolic or flat-topped $\mathrm{CO}$ emission profiles. Assuming that the profiles are the result of optically thick envelopes, the mass-loss rates for the stars can be estimated using a formula of Knapp \& Morris (1985), corrected for the different beam characteristics of the telescope and for the higher transition as discussed in Woodsworth et al. (1991):

$$
\dot{M}=4.7 \times 10^{-8}\left(T_{A}^{*} / \mathrm{K}\right)\left(V_{e} / \mathrm{km} \mathrm{s}^{-1}\right)^{2}\left(D / \mathrm{kpc}^{2} M_{\odot} \mathrm{yr}^{-1}\right.
$$

where $T_{A}^{*}$ is the antenna temperature corrected to outside Earth's atmosphere, $V_{e}$ is the envelope expansion velocity, and $D$ is the distance. The derived mass-loss rates as a function of distance are given in Table 2. This formula assumes a $\mathrm{CO}$ to $\mathrm{H}_{2}$ abundance ratio of $8 \times 10^{-4}$.

Distance estimates to any of these sources are difficult to obtain. Kinematic distances can be found for 11 of the 17 sources in Table 2, but the radial velocities of AFGL 3068 and IRAS $21282+5050$, which are probably close by, indicate significant deviations from the Galactic rotation curve so the values are probably not too accurate. The alternative is to assume a constant bolometric or infrared luminosity and derive a distance from the IRAS data, as is done by Jura \& Kleinmann (1990). Since the intrinsic luminosities of AGB stars range over a factor of 10 , this method can also have large errors. The Jura \& Kleinmann (1990) distances are always much smaller than the kinematic distances, often by more than a factor of 2, but even these distances lead to mass-loss rates for $5.4 \times 10^{-6}$ to $3.5 \times 10^{-4} M_{\odot} \mathrm{yr}^{-1}$ with most of the values near $2 \times 10^{-5} M_{\odot} \mathrm{yr}^{-1}$ and only one derived mass-loss rate $<1 \times 10^{-5} M_{\odot} \mathrm{yr}^{-1}$. Thus we take $2 \times 10^{-5} M_{\odot} \mathrm{yr}^{-1}$ to be a typical mass-loss rate for these sources, but this estimate is very uncertain.

For AFGL 3068, IRAS $04530+4427$, and IRAS

TABLE 2

Derived PARAMETERS

\begin{tabular}{|c|c|c|c|c|c|}
\hline $\begin{array}{c}\text { IRAS Name } \\
\text { (1) }\end{array}$ & $\begin{array}{c}\dot{M} / D^{2 \mathrm{a}} \\
\left(M_{\odot} \mathrm{yr}^{-1} / \mathrm{kpc}^{2}\right) \\
(2)\end{array}$ & $\begin{array}{l}\text { Observed Flux } \\
\left(\text { ergs } \mathrm{cm}^{-2} \mathrm{~s}^{-1}\right) \\
\text { (3) }\end{array}$ & $\begin{array}{c}4 \pi F(D / \mathrm{kpc})^{2} \\
\left(100 L_{\odot}\right) \\
(4)\end{array}$ & $\begin{array}{c}\beta \\
(5)\end{array}$ & $\begin{array}{c}\text { Nature } \\
\text { (6) }\end{array}$ \\
\hline $00210+6221$. & $2.6(-6)$ & $1.7(-8)$ & 5 & 4.0 & PN \\
\hline $01144+6658 \ldots$ & $1.4(-5)$ & $5.2(-8)$ & 16 & 8.5 & PPN \\
\hline $02345+5422 \ldots$ & $5.3(-6)$ & $1.2(-8)$ & 4 & 12.9 & ... \\
\hline $03313+6058$ & $2.5(-6)$ & $1.1(-8)$ & 3 & 5.1 & $\ldots$ \\
\hline $04530+4427 \ldots \ldots \ldots \ldots$ & $7.2(-6)$ & $3.3(-8)$ & 10 & 7.2 & C star? \\
\hline $06582+1507 \ldots \ldots \ldots \ldots$ & $2.5(-6)$ & $1.4(-8)$ & 4 & 4.6 & $\ldots$ \\
\hline $18464-0656 \ldots \ldots \ldots \ldots$ & $3.6(-6)$ & $3.1(-8)$ & 10 & 3.1 & ... \\
\hline $19075+0921 \ldots \ldots \ldots \ldots$ & $9.5(-6)$ & $4.8(-8)$ & 15 & 6.8 & $\ldots$ \\
\hline $19454+2920 \ldots \ldots \ldots \ldots$ & $3.1(-6)$ & $2.0(-8)$ & 6 & 4.5 & PPN \\
\hline $19480+2504 \ldots \ldots \ldots \ldots$ & $5.8(-6)$ & $1.4(-8)$ & 4 & 17.9 & PPN \\
\hline $19594+4047 \ldots \ldots \ldots \ldots$ & $1.0(-5)$ & $1.4(-7)$ & 48 & 2.0 & C star \\
\hline $21282+5050 \ldots \ldots \ldots \ldots$ & $5.6(-5)$ & $2.1(-8)$ & 7 & 76.6 & PN \\
\hline $21318+5631 \ldots \ldots \ldots \ldots$ & $1.6(-5)$ & $1.0(-7)$ & 29 & 5.5 & $\ldots$ \\
\hline $21489+5301 \ldots \ldots \ldots \ldots$ & $1.8(-5)$ & $4.2(-8)$ & 13 & 19.0 & C star? \\
\hline $22303+5950 \ldots \ldots \ldots \ldots$ & $4.4(-6)$ & $2.2(-8)$ & 6 & 7.6 & $\cdots$ \\
\hline $23166+1655 \ldots \ldots \ldots \ldots$ & $2.4(-5)$ & $2.6(-7)$ & 77 & 2.4 & C star \\
\hline $23321+6545 \ldots \ldots \ldots \ldots$ & $5.8(-6)$ & $1.2(-8)$ & 6 & 9.0 & PPN \\
\hline
\end{tabular}

a The mass-loss rates (col. [2]) are derived from the formula of Knapp \& Morris 1985 which may not be applicable to PPNs and PNs.

${ }^{\mathrm{b}}$ See $\S 5$. 
$06582+1507$ mass-loss rates have been estimated from a dustcontinuum radiative-transfer model fit to the observed energy distribution (see Paper I). These mass-loss values scale differently with distance than do the $\mathrm{CO}$ estimates, so in principle a distance can be derived by requiring agreement between the two values. However, the model fits in Paper I for all three of these sources indicate a mass-loss rate that is increasing with time. Since the $\mathrm{CO}$ mass-loss rates are based on a constant mass-loss assumption, one would expect discrepancies between the mass-loss rates calculated by the two different methods.

From the dust continuum models, we obtain $\dot{M} / D$ of $3.3 \times 10^{-6}$, and $2.3 \times 10^{-5} M_{\odot} \mathrm{yr}^{-1} \mathrm{kpc}^{-1}$ for AFGL 3068, IRAS $04530+4427$, and IRAS $06582+1507$, respectively. Comparison of these values with those in Table 2 suggests that for AFGL 3068 the two estimates are similar and would agree if $D$ is $1.4 \mathrm{kpc}$. For IRAS $06582+1507$ the values are quite different and agreement would require a distance of $9.3 \mathrm{kpc}$. In both cases the distances found this way are almost certainly too large: they imply distances from the Galactic plane $(z)$ of $910 \mathrm{pc}$ and $1.4 \mathrm{kpc}$ and luminosities of 18,500 and $40,800 L_{\odot}$ for AFGL 3068 and $06582+1507$, respectively. If the mass-loss rates are not constant, then it is likely that all of the mass-loss rates estimated in Table 2 are systematically underestimated due to the inappropriateness of the Knapp \& Morris formula.

IRAS $04530+4427$ is an exception to the above statement because the dust mass-loss rate is lower than the CO mass-loss rate. For the two estimates to agree, a distance of about $500 \mathrm{pc}$ is required. However, this would result in a total luminosity of only $350 L_{\odot}$. It is possible that the grain formation in the circumstellar shell is much less efficient than was assumed in deriving the gas mass-loss rate from the dust shell radiative transfer model in Paper I, as might occur because the $\mathrm{C} / \mathrm{O}$ abundance ratio is lower for this source than for typical extreme carbon stars. If the actual distance to this source is 2 $\mathrm{kpc}$, the dust-to-gas ratio would have to be a factor of 4 lower than had been assumed in Paper I. There is no allowable kinematic distance for this source, but estimates from the bolometric of infrared flux give a distance of order $2.5 \mathrm{kpc}$ for this source, with large uncertainties.

A study of S-type stars by Jura (1988) suggests that the mean $\left[\mathrm{CO} / \mathrm{H}_{2}\right]$ value for four S-stars is about a factor of 2 lower than the mean value for carbon stars. This may account for about half of the discrepancy, but the dust formation rate must still be less efficient than usual to allow agreement.

\section{INDIVIDUAL SOURCES}

Some of these sources have previously been suggested as extreme carbon star candidates either due to the lack of LRS spectral features of because of $11.3 \mu \mathrm{m} \mathrm{SiC}$ emission. In most cases there has been no direct observation of the stellar photosphere for conventional spectral classification, except for AFGL 3068 and IRAS 19594+4047 = AFGL 2494 where the underlying carbon star is directly detected with near-infrared spectrophotometry (Merrill \& Stein 1976). In the following sections we discuss the previous observations of these sources and some implications of our observations.

\subsection{Featureless or Weak $\mathrm{SiC}$ Sources with Color Temperatures of 300-450 K (Group 1)}

IRAS $00210+6221 .-\mathrm{CO}(J=1 \rightarrow 0)$ emission was detected by Arquilla, Leahy, \& Kwok (1987). This object was found to have a bright $(V=16.5)$ optical counterpart and was suggested as a PN (Hrivnak, Kwok, \& Boreiko 1985). The mid-infrared photometry by Kwok et al. (1987) shows a featureless continuum which is consistent with the IRAS LRS spectrum extracted later at the University of Calgary IRAS Data Analysis Facility. The excess in the visible part of the spectrum (Paper I) is likely to be due to nebular emission, and the object is probably a very young $\mathrm{C}$-rich $\mathrm{PN}$.

IRAS $01144+6658$ (AFGL 190).-This source has been observed several times previously in molecular lines. A previous $\mathrm{CO}(J=2 \rightarrow 1)$ observation is given by Zuckerman \& Dyck (1986a), giving $V_{\mathrm{LSR}}$ and $V_{e}$ values that are nearly identical to those we have observed. $\operatorname{HCN}(J=2 \rightarrow 1)$ was marginally detected by Zuckerman \& Dyck (1986b). A combination of the ground-based near-infrared photometry and the IRAS data shows that its energy distribution is well fitted by a $313 \mathrm{~K}$ blackbody (Paper I)

IR AS $02345+5422$. - There appear to be no previous infrared or molecular line observations of this source besides the IRAS data.

IRAS $03313+6058$.- Other than the search for $\mathrm{OH}$ maser emission (Galt, Kwok, \& Frankow 1989), there do not appear to be any previous observations of this source besides the IRAS data.

IRAS $04530+4427$. - This source has been classified as an infrared carbon star based on the weak $11.3 \mu \mathrm{m} \mathrm{SiC}$ emission feature in its LRS spectrum. Chan \& Kwok (1990) derive a mass-loss rate of $5.8 \times 10^{-6} M_{\odot} \mathrm{yr}^{-1}\left(D / \mathrm{kpc}^{-1}\right.$ based on the IRAS data. CO observations have been made by Zuckerman \& Dyck (1986a, b; $J=1 \rightarrow 0$ ), and Woodsworth et al. (1991, $J=2 \rightarrow 1)$. HCN $(J=1 \rightarrow 0)$ observations have been made by Nguyen-Q-Rieu et al. (1987). Infrared photometry of this object was reported in Paper I.

IR AS $06582+1507$. - This source has no previous observations beside the IRAS survey, an unsuccessful $\mathrm{OH}$ maser observation by Lewis, Eder, \& Terzian (1990), and the infrared observations from Paper I.

IRAS 18464-0656 (AFGL 2256).--Le Squeren et al. (1992) searched and failed to detect $\mathrm{OH}$ emission from this object. There are no other ground-based observations.

IRAS $19075+0921$. - This source has no previous observations aside from the IRAS data. Our JCMT spectrum (see Fig. 3 ) is contaminated by several narrow interstellar, lines, including some in the reference beam. The line contamination makes the derived line parameters for this source in Tables 1 and 2 somewhat uncertain. The LRS spectrum for $19075+0921$ has what appears to be an interstellar silicate absorption feature superimposed on the carbon star spectrum. From the strength of the $10 \mu \mathrm{m}$ absorption feature, we estimate an $A_{v}$ of $3.6 \mathrm{mag}$, assuming the extinction law of Rieke \& Lebofsky (1985). This translates to a distance of $\sim 4 \mathrm{kpc}$. If this source is indeed that far away, its mass-loss rate must be extremely high.

IRAS $19238+1159$. - There is no previous observation for this object other than the discovery by IRAS. Like $19075+0921$, the absorption feature at $10 \mu \mathrm{m}$ is probably interstellar, suggesting a large distance for this object.

IRAS 19594+4047 (AFGL 2494).-This source is known to be a carbon star from near-infrared spectrophotometry (Merrill \& Stein 1976). A mass-loss rate of $1.7 \times 10^{-6} M_{\odot}$ $\mathrm{yr}^{-1}(D / \mathrm{kpc})^{-1}$ was derived by Chan \& Kwok (1990) from the infrared data. It has been observed in $\mathrm{CO}(J=1 \rightarrow 0)$ and HCN by Zuckerman \& Dyck (1986a, b). Near-infrared photometry by Jones et al. (1990) indicates that this star has a $680^{\circ}$ pulsation period.

IRAS $21318+5631$. - This source has near-infrared photometric observations by Gaylard et al. (1989). Previous CO 
$(1 \rightarrow 0)$ observations are given by Zuckerman, Dyck, \& Claussen (1986) and Lindquist et al. (1988).

IRAS $21489+5301$. - This source is known to be an infrared carbon star because of the $11.3 \mu \mathrm{m} \mathrm{SiC}$ feature in its LRS spectrum (Chan \& Kwok 1990). The mass-loss rate derived from model fitting to the infrared data is $7.1 \times 10^{-6} M_{\odot} \mathrm{yr}^{-1}$ $(D / \mathrm{kpc})^{-1}$. The object was observed in $\mathrm{CO}(2 \rightarrow 1)$ by Zuckerman \& Dyck (1986a), and in CO $(1 \rightarrow 0)$ and HCN $(1 \rightarrow 0)$ by Nguyen-Q-Rieu et al. (1987).

IR AS $22303+5950$.- This source is known to be an extreme carbon star due to the $\mathrm{SiC}$ feature in its LRS spectrum. It was observed in CO $(1 \rightarrow 0)$ and HCN $(1 \rightarrow 0)$ by Nguyen-Q-Rieu et al. (1987) but otherwise seems to have no data aside from the IRAS observations.

\subsection{Low-Temperature (Group 2) Sources}

IRAS $19454+2920$. - This is one of the first IRAS sources found to possess a low-temperature featureless continuum (Kwok et al. 1987). The dust component is well represented by a $190 \mathrm{~K}$ blackbody, but in the near-infrared region it shows an excess of a $\sim 3500 \mathrm{~K}$ blackbody (Kwok, Hrivnak, \& Volk 1990). These properties suggest that $19454+2920$ is a PPN which recently left the AGB. CO emission was first detected by Arquilla, Leahy, \& Kwok (1987), and further observations were also obtained by Likkel et al. (1987, 1991).

IRAS $19480+2504$. - This source was observed in CO by Likkel et al. (1991). Near-infrared photometry (Kwok, Langill, \& Hrivnak 1992) shows excess over the dust continuum, suggesting that this is a PPN.

IRAS $23321+6545$. - This source was observed in $\mathrm{CO}$ by Likkel et al. $(1987,1991)$. As in the case of $19454+2920$, they obtained a smaller $V_{e}$ value than we did. This object was also detected at $\lambda 1.3 \mathrm{~mm}$ by Walmsley et al. (1991).

The CO detection of three of the four sources with lowtemperature, featureless IRAS LRS spectra indicates that these sources have expanding circumstellar envelopes similar to those of the extreme carbon star candidates. However, it is puzzling that IRAS $19159+1556$ was not detected. As can be seen from Figure 1, this has the brightest LRS spectrum of the four objects. There is another peculiarity of IRAS $19159+1556$ compared with the other sources. The IRAS broad-band photometry data for this object is badly inconsistent with the LRS spectrum. The measured flux densities for IRAS $19159+1556$ are $1.92 \mathrm{Jy}$ at $12 \mu \mathrm{m}$ and $1.08 \mathrm{Jy}$ at $25 \mu \mathrm{m}$. For comparison, the values for IRAS $23321+6545$ are $13.66 \mathrm{Jy}$ at $12 \mu \mathrm{m}$ and 85.61 Jy at $25 \mu \mathrm{m}$. If one assumes that the $25 \mu \mathrm{m}$ flux density and the LRS flux density near $23 \mu \mathrm{m}$ should be proportional for these two objects, since their spectral shapes are very similar, the resulting estimated $25 \mu$ m flux density for IRAS $19159+1556$ is $143 \mathrm{Jy}$, or more than 100 times larger than the observed value. The discrepancy at $12 \mu \mathrm{m}$ is also quite large.

Such a disagreement between the LRS data and the photometric data is very unusual. The LRS had an instantaneous field of view that was $6^{\prime}$ by $15^{\prime}$, compared with about 0.5 square for the photometric observations at 12 and $25 \mu \mathrm{m}$. If the source is significantly extended this might explain the brightness disagreement, for this is occasionally observed in other cases where the source is known to be more extended than the size of the IRAS photometric beam, but there was no hint that the source is extended in the photometric observations. The data scans matched the point-source template very well at 12 and 25 $\mu \mathrm{m}$ according to the IRAS Point Source Catalog, nor do the LRS data scans show any unusual features, as would be expected if the source were of similar size to the LRS aperture.
In order to resolve these questions, we sent a query to IPAC to check the source for evidence of extendedness. There are indications in the survey scans that $19159+1556$ was confused with a near-field source, probably an asteroid. This would explain the flux density discrepancy and the lack of $\mathrm{CO}$ emission in the object.

The three detected group 2 sources do not seem to show a correlation between the CO line strength and the IRAS photometry values, or between the derived $\dot{M} / D^{2}$ values and the $I R A S$ photometry values. These three sources are all brighter than IRAS $21282+5050$ at 25,60 , and $100 \mu \mathrm{m}$ (the $12 \mu \mathrm{m}$ flux density from IRAS $21282+5050$ is strongly affected by the unidentified infrared emission features which seem to produce almost all of the flux at wavelengths shorter than $18 \mu \mathrm{m}$ ) but have much weaker $\mathrm{CO}$ emission. However, these sources appear to have a larger $\mathrm{CO}$ emission to infrared luminosity ratio than do the extreme carbon star candidates. We will further address this point in $\S 5$.

The referee suggests that the nondetected objects could be $\mathrm{OH} / \mathrm{IR}$ stars. We consider this unlikely because $(a)$ the $I R A S$ spectra and colors of these objects are quite different from $\mathrm{OH} / \mathrm{IR}$ stars and $(b) \mathrm{OH}$ emission has been searched for but not found in $19238+1159$ and in three of the four group 2 sources (Lewis et al. 1990; Likkel 1989).

\subsection{The Carbon-rich PN IRAS $21282+5050$}

For comparison, we have also observed the recently discovered C-rich PN IRAS $21282+5050$. This object shows PAH features in both the near- and mid-infrared (de Muizon et al. 1986) and is found to have an emission-line spectrum similar to that of PN. From the nebular spectrum, the central star is classified as 07(f)-[WC 11] (Cohen \& Jones 1987). It is likely that the featureless dust component represents the remnant of the circumstellar envelope of an extreme carbon star, and the PAH features are excited by the central star which has recently become hot enough to ionize the circumstellar envelope.

$\mathrm{CO}$ emission from $21282+5050$ was detected by Likkel et al (1988). Our CO results for IRAS $21282+5050$ are not totally in agreement with the previous results of Likkel et al. (1991). In ${ }^{12} \mathrm{CO}(2-1)$ they report a maximum brightness temperature of $15.1 \mathrm{~K}, V_{\mathrm{LSR}}=18.8 \mathrm{~km} \mathrm{~s}^{-1}$ and $V_{e}=15.8 \mathrm{~km} \mathrm{~s}^{-1}$. Our $V_{\mathrm{LSR}}$ value of +15.7 differs slightly from their value of $18.8 \mathrm{~km} \mathrm{~s}^{-1}$, but our velocity resolution is better than theirs so our value is likely more accurate. Also, our $V_{e}$ value is somewhat higher than their value. Their Figure 1 shows the observed $\mathrm{CO}$ profiles, which look very similar to the profile we obtained, so the disagreement in $V_{e}$ is probably due to the use of line profile fits by Likkel et al. (1988). They consider there to be "high-velocity wings" on the $\mathrm{CO}$ profile, although their Figure 1 seems to show these wings as extending only of order $5 \mathrm{~km} \mathrm{~s}^{-1}$ beyond the main line component. Their brightness temperature value and our brightness temperature value are consistent if the $\mathrm{CO}$ emission has a size of $11^{\prime \prime}$, resulting in a factor of 4 greater beam dilution for the JCMT observations compared to IRAM which has a diameter twice that of JCMT.

The mass-loss rate deduced from the $\mathrm{CO}$ observations of Likkel et al. (1988) is $1.4 \times 10^{-5} M_{\odot} \mathrm{yr}^{-1}(D / \mathrm{kpc})^{2}$, which is lower than our value by a factor of 3.7. Even if we adopt their value of $V_{e}$, the above rates still differ by a factor of 2 .

$21282+5050$ also has an energy distribution which is very unusual for a PN (Kwok et al. 1982). Most of the energy is emitted from the dust component. Based on the analysis of Zhang \& Kwok (1991), the fraction of energy emitted by the 
dust component is no more than $50 \%$ even for very young PN. The properties of $21292+5050$ can only be understood if it has a massive nucleus $\left(>1 M_{\odot}\right)$ which allows it to evolve across the H-R diagram before the dust envelope dissipates. In this regard, it is very similar to NGC 7027.

Optical and radio imaging of $21292+5050$ show a size of $\sim 5^{\prime \prime}$ (Kwok et al. 1992; Likkel et al. 1992). The CO-emitting region is resolved by the Nobeyama Millimeter Array, and the CO emission is found to extend over 15" (Shibata et al. 1989). This suggests that the nebula is ionization bounded.

\subsection{HCN Observations}

Three sources were observed in the $\operatorname{HCN}(J=3 \rightarrow 2)$ transition (see Table 1). Both AFGL 3068 and IRAS 01144+6658 have previously been observed in the $\mathrm{HCN}(2 \rightarrow 1)$ transition. The HCN $(2 \rightarrow 1)$ observations gave line brightness temperatures of $0.55 \mathrm{~K}$ for AFGL 3068 using a 42" FWHP beam and $0.07 \mathrm{~K}$ for IRAS $01144+6658$ using a $69^{\prime \prime}$ FWHP beam (Sopka et al. 1989; Zuckerman \& Dyck 1986b). Expressed as ratios to the $\mathrm{CO}(1 \rightarrow 0)$ line, the line strengths are 0.34 and 0.13 , respectively. The line $V_{\mathrm{LSR}}$ and $V_{e}$ values from these observations are similar to those we derived from the $3 \rightarrow 2$ transition. The line ratios for the higher transitions appear to be slightly larger than for the lower transitions. The ratio of $\mathrm{HCN}$ $(3 \rightarrow 2)$ and $\mathrm{CO}(2 \rightarrow 1)$ peak line intensities is 0.46 for AFGL $3068,0.30$ for IRAS $01144+6658$, and can be crudely estimated as less than 0.05 for IRAS $21282+5050$. In terms of integrated line intensity the ratios are 0.40 for AFGL 3068 and 0.24 for IRAS $01144+6658$.

While Jura (1991) has noted an increase of HCN line strengths with the expansion velocities in AGB stars, this correlation does not extend to PPNs and PNs, which generally have higher expansion velocities. As noted above, the HCN line strengths in fact decreases beyond the AGB. The absence of the HCN in $21282+5050$ can be understood by the fact that the $\mathrm{HCN}$ line requires higher excitation and is generally located in the inner parts of the circumstellar envelope. In the case of $21282+5050$, the inner part of the envelope is already ionized (out to $\sim 5^{\prime \prime}$ ), and therefore no HCN can be detected.

However, the very young PN NGC 7027 is known to show $\mathrm{HCN}(2 \rightarrow 1)$ emission at about $5 \%$ of the $(1 \rightarrow 0)$ line strength (Sopka et al. 1989), which makes its HCN emission more than 15 times stronger than for IRAS $21282+5050$. Both are C-rich PNs as they both show PAH features. NGC 7027 has a much hotter central star $(170,000$ vs. $30,000 \mathrm{~K})$, but in comparison to $21282+5050$, its ionized region $\left(10^{\prime \prime}\right)$ is relatively small compared to its molecular envelope (3'). It is clear that NGC 7027 has a smaller dynamical age than $21282+5050$, and yet its central star is more evolved. The rapid evolution across the H-R diagram suggests that NGC 7027 has a more massive central star than $21282+5050$.

\subsection{Other Comments}

IRAS $06582+1507$. - This source is unusual among the extreme carbon star candidates because its LRS spectrum is well fitted by a pure graphite (or possibility amorphous carbon) dust shell of very high optical depth (see Paper I). Based upon the model fit, if this source has a luminosity of $\sim 7000 L_{\odot}$ typical of a normal AGB star of lower mass it must be at a distance of $\sim 4 \mathrm{kpc}$. It shows no unusual $\mathrm{CO}$ properties, having a normal ratio of $\mathrm{CO}$ line strength to infrared flux density.

IRAS $21489+5301$. - This source has an anomalously strong CO line for its infrared fluxes. Its line is about a factor of 2 stronger than would be expected from its $60 \mu \mathrm{m}$ flux density if other sources in the sample are used to define a mean ratio.

\section{MOMENTUM AND RADIATIVE FLUXES IN PPNS AND PNS}

The momentum flux-to-luminosity ratio, defined as $\beta=$ $\dot{M} V_{e} c / L_{*}$, generally has a value near unity of AGB stars. However, for a number of PNs and PPNs $\beta$ has been found to exceed unity by a large factor (Knapp \& Morris 1985; Knapp 1986). This has been interpreted to imply an absolute decrease in luminosity during the evolutionary transition between AGB and PN phases (Knapp 1987, 1989). In Table 2, we have evaluated the values of $\beta$ for our program objects. The total emitted flux of the objects (given in col. [3] of Table 2) were estimated by fitting the IRAS data by a blackbody curve. Since the energy distribution of most of the sources are dominated by the low-temperature dust component, these values should be accurate to within $25 \%$. Since the PN $21282+5050$ is likely to be ionization bounded (see $\S 4.3$ ), the leakage of UV flux should be small. We can see that the values of $\beta$ range from 2 for the carbon star $19594+4047$ to 77 for the PN $21282+5050$. The PPNs also have high $(>5) \beta$ values.

The change in the value of $\beta$ by almost two orders of magnitude is difficult to attribute to luminosity change. The luminosity of PPNs and PNs are due to hydrogen shell burning on a electron-degenerate core and should only be dependent on the core mass, which suffers very small change (and certainly does not decrease) during the PPN-PN evolution. The possibility of the large $\beta$ values in PNs and PPNs as the result of bipolar morphology was considered by Hrivnak \& Kwok (1991). Circumstellar disks viewed edge-on could result in an underestimation of the observed flux in the visible part of the spectrum. While this explanation is attractive qualitatively, it is uncertain whether it can explain the quantitative difference. Another possibility is the CO in PNs and PPNs is excited differently than in AGB stars. For example, the dust temperature is lower in PNs and PPNs, which may result in a larger far-infrared flux in the radiative excitation to vibrational states. Another possibility is that the population distribution of the CO molecule in PNs and PPNs concentrates more in the lower rotational states, resulting in higher excitation temperatures of the lower rotational transitions. These two possibilities should be quantitatively evaluated by line transfer models. In either case, the mass-loss rates cannot be derived from the Knapp \& Morris formula, which results in a overestimate of the mass loss rate from PPNs and PNs.

\section{SUMMARY AND CONCLUSIONS}

We have obtained $\mathrm{CO}(J=2 \rightarrow 1)$ observations of 19 low color temperature IRAS sources that show weak $\mathrm{SiC}$ emission or featureless continuum in the 8-22 $\mu \mathrm{m}$ range. Except for the source which was later found to be an astroid, circumstellar $\mathrm{CO}$ emission was detected in 17 of the remaining 18 sources. Five of the 17 are new detections (never detected in CO before), and an additional four were detected in the $J=2 \rightarrow 1$ transition for the first time. The high detection rate supports the idea that all of these sources are either extreme carbon stars or carbon stars which have recently left the asymptotic giant branch.

Using a formula adapted from Knapp \& Morris (1985), the mass-loss rate as a function of distance has been calculated for all of the sources. If the mean source distance is of order 2-3 kpc, which can be crudely estimated from the IRAS data 
assuming a total luminosity appropriate for an AGB star, the mass-loss rates of these objects are $\sim 2 \times 10^{-5} M_{\odot} \mathrm{yr}^{-1}$

As previously noted by Knapp (1987), we find that ratio of the $\mathrm{CO}$ emission strength to photon flux decreases from AGB stars to PPNs to PNs. This ratio $(\beta)$ changes from $\sim 1$ for AGB stars to $\sim 100$ for PNs. This change cannot be the result of - luminosity decrease as suggested by Knapp (1987), and the effect of missing some of the observed flux in a bipolar geometry is also unlikely to adequately explain the magnitude of the difference (Hrivnak \& Kwok 1991). We believe that this change is the result of increasng radiative excitation of $\mathrm{CO}$ as the dust envelope cools in the post-AGB evolution.

$\mathrm{HCN}(3 \rightarrow 2)$ observations were carried out for three objects: AFGL 3068, IRAS 01144+6658, and IRAS $21282+5050$. The $\mathrm{HCN}(3 \rightarrow 2)$ to $\mathrm{CO}(2 \rightarrow 1)$ intensity ratio are $0.46,0.30$, and $<0.05$ for these three objects. This decreasing trend of HCN to $\mathrm{CO}$ ratio is consistent with the evolutionary states of the three sources: late AGB star-PPN-PN. As the density of the envelope decreases as the result of shell detachment, conditions become less and less favorable to the excitation of $\mathrm{HCN}$. When the inner envelope is finally ionized (as in $21282+5050$ ), $\mathrm{HCN}$ can no longer be observed. The possibility of using the HCN to $\mathrm{CO}$ ratio as a tracer of post-AGB evolution should be tested in more PPN and young PN.

The James Clerk Maxwell Telescope is operated by the Royal Observatory Edinburgh on behalf of the Science and Engineering Research Council of the United Kingdom, the Netherlands Organization for Scientific Research, and the National Research Council of Canada. The SIMBAD data base was used to check for relevant bibliographic references. This work is supported in part by NASA Astrophysical Data Program Grant 215-90 and in part by a Natural Sciences and Engineering Research Council operating grant to $\mathrm{S}$. K. The University of Calgary IRAS Data Analysis Facility is supported by a grant from NSERC.

\section{REFERENCES}

Arquilla, R., Leahy, D. A., \& Kwok, S. 1987, MNRAS, 220, 125

Atlas of Low Resolution IRAS Spectra. 1986, IRAS Science Team, prepared by

F. M. Olnon \& E. Raimond (A\&AS, 65, 607)

Chan, S. J., \& Kwok, S. 1990, A\&A, 237, 354

Cohen, M., \& Jones, B. 1987, ApJ, 321, L151

de Muizon, M., Geballe, T. R., d'Hendecourt, L. B., \& Baas, F. 1986, ApJ, 306, L105

Galt, J. A., Kwok, S., \& Frankow, J. 1989, AJ, 98, 2182

Gaylard, M. J., West, M. E., Whitelock, P. A., \& Cohen, R. J. 1989, MNRAS, 236, 247

Herman, J., \& Habing, H. J. 1985, Phys. Rep., 124 (4), 255

Hrivnak, B. J., \& Kwok, S. 1991, ApJ, 368, 564

Hrivnak, B. J., Kwok, S., \& Boreiko, R. T. 1985, ApJ, 294, L113

Iben, I., \& Renzini, A. 1983, ARA\&A, 21, 271

Jones, T. J., Bryja, C. O., Gehrz, R. D., Harrison, T. E., Johnson, J. J., Klebe, D. I., \& Lawrence, G. F. 1990, ApJS, 74, 785

Jura, M. 1988, ApJS, 66, 33

. 1991, ApJ, 372, 208

Jura, M., \& Kleinmann, S. G. 1990, ApJ, 364, 663

Knapp, G. R. 1986, ApJ, 311, 731

. 1987, in Late Stages of Stellar Evolution, ed. S. Kwok \& S. R. Pottasch (Dordrecht: Reidel), 103

. 1989, in IAU Symp. 131, Planetary Nebulae, ed. S. Torres-Peimbert (Dordrecht: Kluwer), 381

Knapp, G. R., \& Morris, M. 1985, ApJ, 292, 640

Knapp, G. R., Phillips, T. G., Leighton, R. B., Lo, K. Y., Wannier, P. G., Wootten, H. A., \& Huggins, P. J. 1982, ApJ, 252, 616

Kwok, S. 1987, Phys. Rep., 156 (3), 111

Kwok, S., Hrivnak, B. J., \& Boreiko, R. T. 1987, ApJ, 312, 303

Kwok, S., Hrivnak, B. J., \& Volk, K. 1990, in from Miras to Planetary Nebulae: Which Path for Stellar Evolution?, ed. M. O. Mennessier \& A. Omont (Gif-sur-Yvette: Editions Frontières), 435
Kwok, S., Langill, P. L., \& Hrivnak, B. J. 1992, in preparation

Lawrence, G., Jones T. J., \& Gehrz, R. D. 1990, AJ, 99, 1232

Le Sequeren, A. M., Sivagnanam, P., Dennefeld, M., \& David, P. 1992, A\&A 254,133

Lewis, B. M., Eder, J., \& Terzian, Y. 1990, ApJ, 362, 634

Likkel, L. 1989, ApJ, 344, 350

Likkel, L., Forveille, T., Omont, A., \& Morris, M. 1988, A\&A, 198, L1

. 1991, A\&A, 246, 153

Likkel, L., Morris, M., Omont, A., \& Forveille, T. 1992, in preparation

Linkel, L., Omont, A., Morris, M., \& Forveille, T. 1987, A\&A, 173, L11

Lindqvist, M., Nyman, L. A., Olofsson, H., \& Winneberg, A. 1988, A\&A, 205, L15

Matthews, H. E. 1991, The James Clerk Maxwell Telescope: A Guide For The Prospective User, Royal Observatory, Edinburgh

Merrill, M., \& Stein, W. A. 1976, PASP, 88, 874

Nguyen-Q-Rieu, Epchtien, N., Troug-Bach, \& Cohen, M. 1987, A\&A, 180, 117

Rieke, G. H., \& Lebofsky, M. J. 1985, ApJ, 288, 618

Shibata, K. M., Tamura, S., Deguchi, S., Hirano, N., Kameya, O., \& Kasuga, T. 1989, ApJ, 345, L55

Sopka, R. T., Olofsson, H., Johnson, L. E. B., Nguyen-Q-Rieu, \& Zuckerman, B. 1989, A\&A, 210, 78

Volk, K., Kwok, S., \& Langill, P. L. 1992, ApJ, 391, 285, (Paper I)

Volk, K., Kwok, S., Stencel, R., \& Brugel, E. 1991, ApJS, 77, 607

Walker, H. J., \& Cohen, M. 1988, AJ, 95, 1801

Walmsley, C. M., Chini, R., Kreysa, E., Steppe, H., Forveille, T., \& Omont, A. 1991, A\&A, 246, 555

Woodsworth, A. W., Kwok, S., Chan, S. J., \& Murowinski, R. 1991, A\&A, 246 447

Zhang, C. Y., \& Kwok, S. 1991, A\&A, 250, 179

Zuckerman, B., \& Dyck, H. M. 1986a, ApJ, 304, 394

. 1986b, ApJ, 311, 345

Zuckerman, B., Dyck, H. M., \& Claussen, M. J. 1986, ApJ, 304, 401 